\title{
Establishing the Interface for G-Bot Monitoring and Controlling System
}

\author{
Dewi Permata Sari ${ }^{1}$, Nyayu Latifah Husni ${ }^{1, *}$, , Fatma Indah Sari ${ }^{1}$, Nurhaida \\ Nurhaida $^{1}$, Yogi Eka Fernandes ${ }^{1}$, Ade Silvia Handayani ${ }^{2}$ \\ ${ }^{l}$ Electronic Engineering Study Program, Electrical Engineering Department, Sriwijaya State Polytechnic \\ ${ }^{2}$ Telecommunication Engineering Study Program, Electrical Engineering Department, Sriwijaya State \\ Polytechnic \\ *Corresponding author.Email: nyayu_latifah@polsri.ac.id
}

\begin{abstract}
Garbage is a worldwide problem that has not been fully handled, including in Indonesia. As citizens, the human has an obligation to dispose the garbage properly, however, there are so many people who still do not care about this problem. In this situation, The author introduces the Garbage Robot (G-Bot) which can be controlled from further place. This GBot works based on the Internet of Things (IoT) in which it can connect things so that they may operate together. This G-Bot can be controlled using some devices, such as: mobile phone, PC, and laptop. However, how to establish the interface for the G-Bot monitoring and controlling system is still a problem. Therefore, this paper proposes the interface design for the G-Bot. Using this interface, it is hoped that the G-Bot can be monitored and controlled using the user's devices from further place. The G-Bot may go from one place to another place using speech recognition, human followers, and user manuals instructions that have been integrated in the devices. In addition, the interface design in this proposed method also includes the menu of the environmental monitoring, such as temperature, humidity, and air quality.
\end{abstract}

Keywords:Garbage, G-Bot, IoT, Environmental Monitoring, Controlling, Human Follower, Speech Recognition

\section{INTRODUCTION}

Garbage is a material that comes from sources resulting from human or natural activities which is a major global problem and has not been completely resolved [1], [2], including in Indonesia. Many people still is not aware of the effect of the garbage to the health [3]-[6]. The habit of throwing the garbage to the improper place poses a serious threat to human life [7][10] .

Actually, the habit of disposing garbage to its place is the obligation of all of the citizens. However, the habits are sometimes still be constrained by human limitations. Therefore, an effective, efficient and innovative way should be made to help the community in disposing the waste. In this research, garbage robots (GBots) that provide organic and inorganic garbage compartmnets are introduced. The G-Bot also provides an indicator if the waste capacity is almost full. In addition, it also displays the information of the temperature, humidity, and the air quality.

The G-Bot works based on the Internet of Things (IoT). It is designed to increase the efficiency and the effectiveness in controlling G-Bot remotely. Through this system, the G-Bot can be controlled through android software. It can be moved from one place to another either through speech recognition, human followers, or user manuals commands. The G-Bot also provides the information of temperature $\left({ }^{\circ} \mathrm{C}\right)$, humidity $(\mathrm{RH})$, air quality (PPM), organic and inorganic waste capacity (\%) via android software and platform that can be monitored in real-time. However, how to establish the interface so that the users device can communicate with the G-bot is still a problem. Thus, this paper proposes the interfaces that can be applied to the G-bot.

\section{METHODOLOGY}

The G-bot is designed using some electronic components as shown in Figure 1. The voltage source comes from the Battery, which supplies all components, except for the Raspberry Pi 4, which uses a PowerBank with a voltage of $3 \mathrm{~V}$. The input sensors from the G-Bot are Ultrasonic HC-SR04 sensor, DHT22 sensor, MQ-8 sensor, Compass sensor HMC5883L, TCS3200 color sensor and Webcam. While the outputs of the G-Bot are 
a 20x4 LCD display, PG45 Motor and MG996 Servo. The input sensor value data will be read by the Arduino Mega 2650 microcontroller and sent to the Raspberry Pi 4. Then, the collected data is sent in real-time using an internet connection to the data cloud, which in this case it is the hosting. Then, it will be displayed on the web interface and mobile software phone. In this case, the access for monitoring can be done through devices, such as: mobile phones, laptops and Personal Computers (PCs) that have a browser installed and connected to the internet. As for controlling the Garbage Robot (G-Bot), the mobile phone must have a G-Bot application installed and connected to the internet, so that it can monitor the Garbage Robot (G-Bot) anytime and anywhere.

In the G-Bot monitoring block diagram, Figure 2, there are inputs in the IoT device, namely temperature $\left({ }^{\circ} \mathrm{C}\right)$ and humidity $(\mathrm{RH})$, DHT-22 sensor, air quality (PPM), MQ-8 sensor, the capacity of organic and inorganic garbage(\%), and sensor HC-SR04. All this input data will be read by the Arduino Mega 2650 microcontroller and sent to the Raspberry Pi 4 using serial communication. In this case the Raspberry Pi 4 which is connected to the internet will directly transmit the collected data in real-time using an internet connection to the data cloud, namely hosting which will be received in the MySQL database and web interface every three seconds. In order to transfer data and interact, the JavaScript Object Notation (JSON) format in the PHP and Java programming languages is used. Then, Application Programming Interface (API) software is also used to integrate two applications so that they can be connected to each other (which in this case is the web interface and mobile phone software, and Ajax. This is done so that data can enter in real-time on the web interface without having to reload the page. Users can monitor through the G-Bot application and the website with the www.g-bot.id domain

The controlling of the G-Bot can be processed using 3 methods, i.e., speech recognition, human follower, and user manual. In the G-Bot application, the user gives the command, in which it can enter the data cloud and be sent to the Raspberry Pi 4 via an internet connection which is then forwarded to the Arduino Mega 2560 and the G-Bot will perform the action of changing classes according to the command.

The user can move the robot from class one to class thirteenth class using the speech recognition method through human voice commands by detecting the last word. If it succeessful, the success alert appears. If the class serial number when ordered is at the beginning or the middle word and the mention of the class number more than thirteen, then it will appear the alert "failed".

In this research, the G-Bot can move using human follower in which the control of the G-Bot which moves to its destination can be estaclished by following the human objects. Besides that, the rovot navigation can be set using the user manual. It is a method of changing classes by clicking on the destination class button. When the command is successful with the indicator on the hosting, namely by entering data on the MySQL database and on the Raspberry Pi 4 which is connected to a router that is connected to the internet, it will give the robot a command to move to the destination class. In order to transfer data or interact, the JavaScript Object Notation (JSON) format in the Java programming language is used. Then, the Application Programming Interface (API) software to integrate the two applications can be connected to each other.

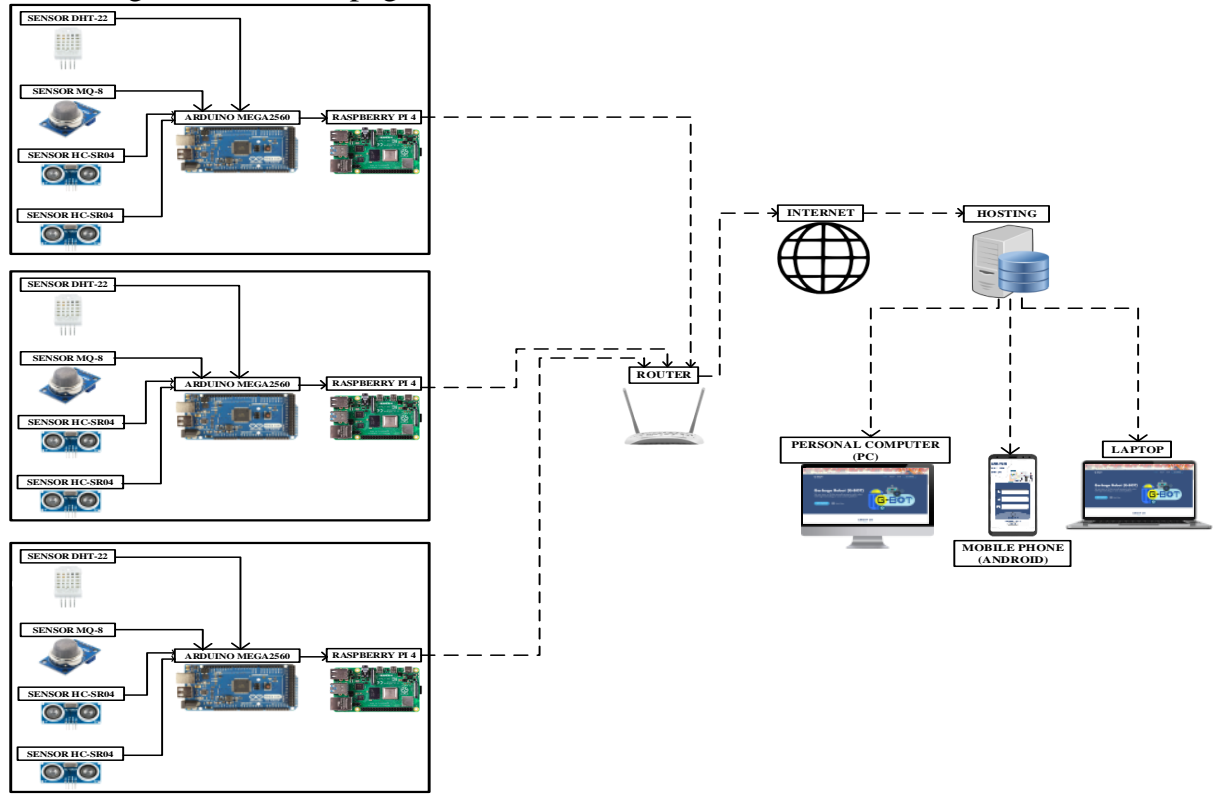

Figure 1 Block Diagram of G-Bot monitoring 

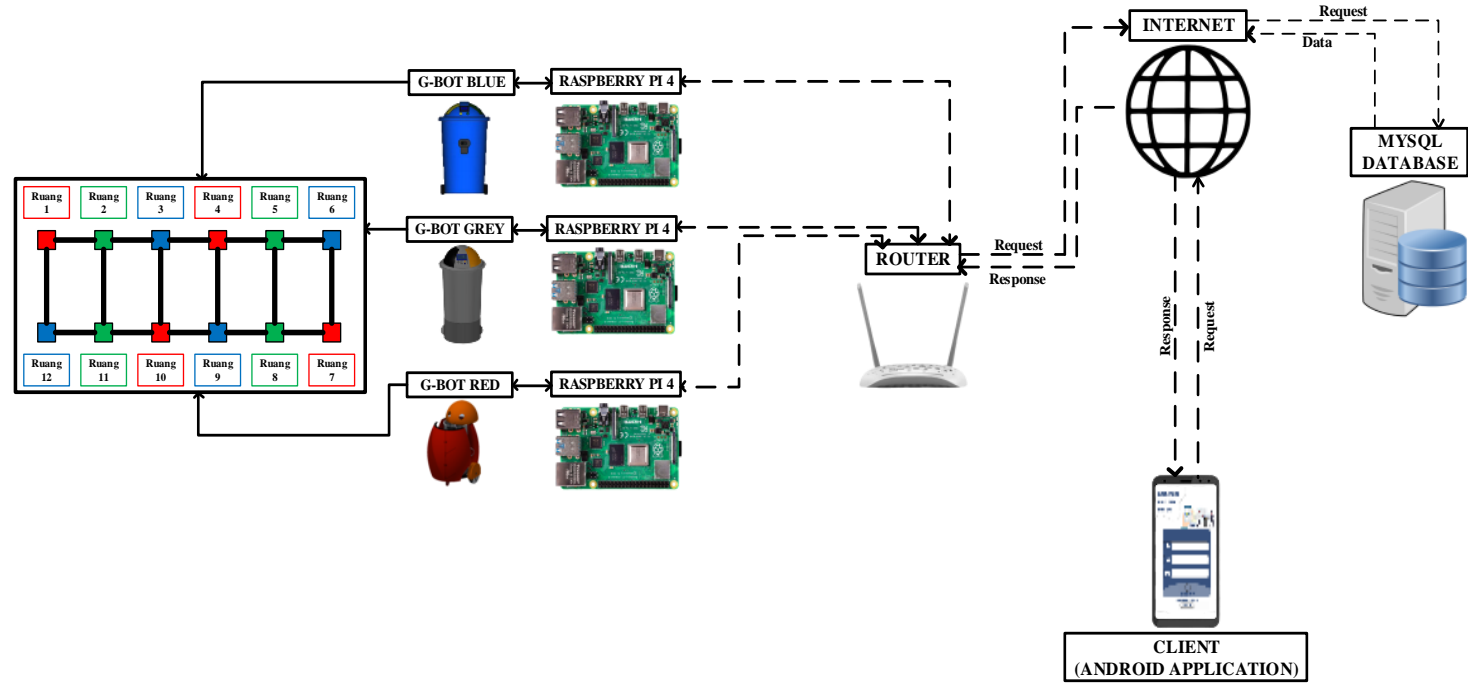

Figure 2 Block Diagram of G-Bot Controlling

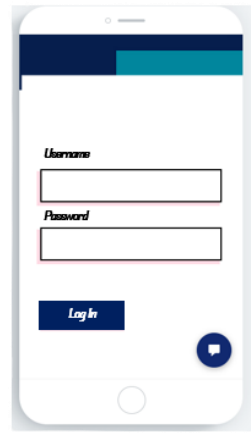

(a) Login

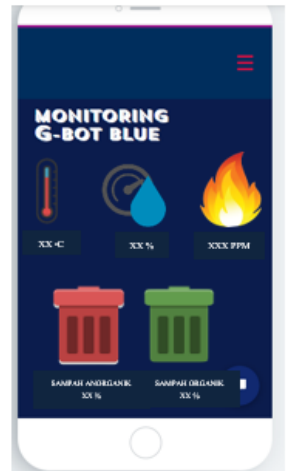

(f) Monitoring G-Bot Blue

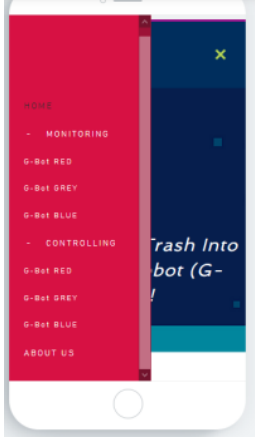

(b) Dashboard

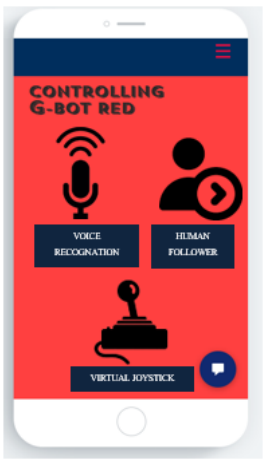

(g) Controlling GBot Red

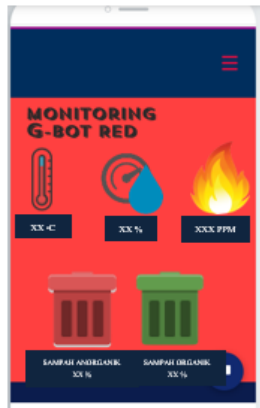

(c) Monitoring G-Bot Red

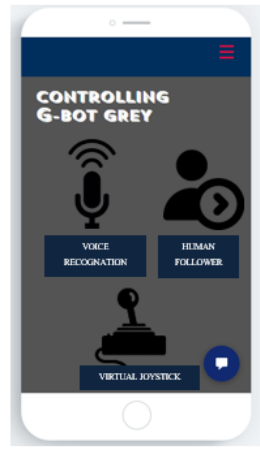

(h) Controlling G-Bot Grey

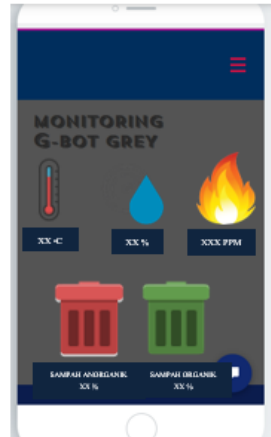

(d) Monitoring G-Bot Grey

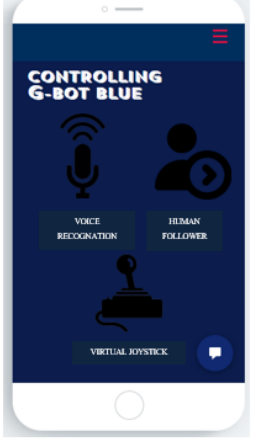

(i) Controlling G-Bot Blue

Figure 3 Interface Design on Mobile phone 


\section{PRPOSED INTERFACE}

The proposed interface is shown in Figure $\mathbf{3}$ and Figure 4. In web interface design for G-Bot monitoring, the design uses monitoring and controlling features for the three robots. The monitoring consists of temperature, humidity, air quality, organic waste capacity and inorganic waste capacity. Then the controlling robot can be conducted through speech recognition, human followers and user manuals commands.

\section{CONCLUSIONS}

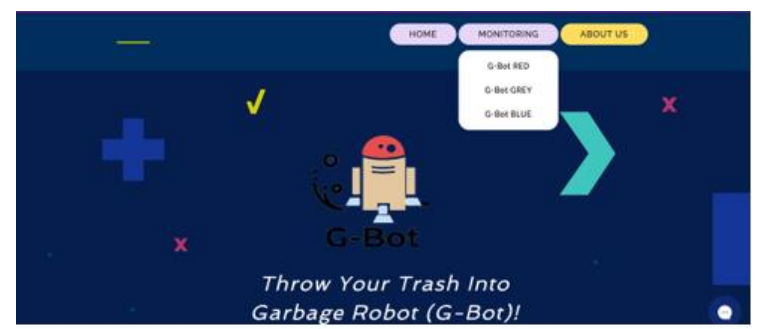

(a) Dashboard

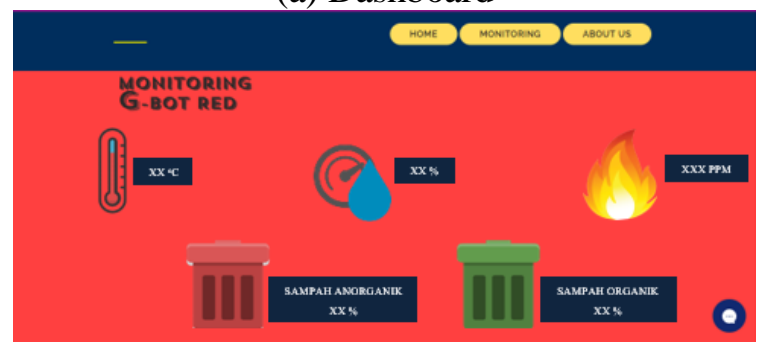

(b) Monitoring G-bot Red

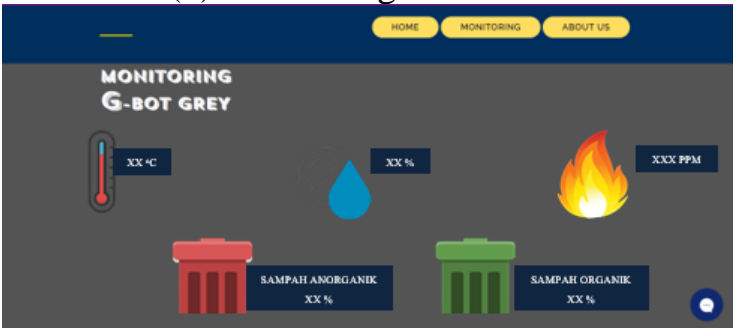

(c) Monitoring G-bot Grey

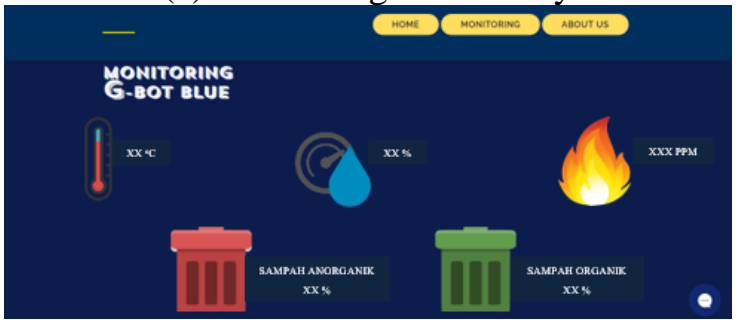

(d) Monitoring G-bot Blue

Figure 4 Interface Design on the Web

The interface of the G-Bot has been established well. It can be connected to the mobile phone and also the computers, or laptop. The controlling of the G-Bot can be conducted using speech recognition system, human follower, and user manual that can be controlled trough the mobile phone interface. While the interface in the monitoring system of the mobile phone, PC, and also laptop, can display the status of temperature, humidity, air quality, organic and inorganic capacity of the garbage.

\section{REFERENCES}

[1] A. Silva, M. Rosano, L. Stocker, and L. Gorissen, "From waste to sustainable materials management: Three case studies of the transition journey," Waste Manag., pp. 1-11, 2016, doi: 10.1016/j.wasman.2016.11.038.

[2] K. Boonrod, S. Towprayoon, S. Bonnet, and S. Tripetchkul, "Enhancing organic waste separation at the source behavior: A case study of the application of motivation mechanisms in communities in Thailand," "Resources, Conserv. Recycl., vol. 95, pp. 77-90, 2015, doi: 10.1016/j.resconrec.2014.12.002.

[3] P. I. Plaza and S. A. Lambertucci, "How are garbage dumps impacting vertebrate demography, heath, and conservation?," Glob. Ecol. Conserv., vol. 12, pp. 920, 2017, doi: 10.1016/j.gecco.2017.08.002.

[4] T. Efferth and N. W. Paul, "Threats to human health by great ocean garbage patches," Lancet Planet. Heal., vol. 1, no. 8, pp. e301-e303, 2017, doi: 10.1016/s2542-5196(17)30140-7.

[5] G. Li, H. Sun, Z. Zhang, T. An, and J. Hu, "Distribution profile, health risk and elimination of model atmospheric SVOCs associated with a typical municipal garbage compressing station in Guangzhou, South China," Atmos. Environ., vol. 76, pp. 173-180, 2013, doi: 10.1016/j.atmosenv.2012.06.027.

[6] J. L. Domingo and M. Nadal, "Domestic waste composting facilities: A review of human health risks," Environ. Int., vol. 35, no. 2, pp. 382-389, 2009, doi: 10.1016/j.envint.2008.07.004.

[7] N. T. Xun, "Garbage Bin Monitoring for Smart Residence," Universiti Tunku Abdul Rahman, 2018.

[8] N. L. Husni, Robi, E. Prihatini, Nurhaida, A. Silvia, and Firdaus, "Garbage Monitoring and Warning System," ICECOS 2019 - 3rd Int. Conf. Electr. Eng. Comput. Sci. Proceeding, pp. 171-175, 2019, doi: 10.1109/ICECOS47637.2019.8984545.

[9] N. L. Husni, S. Sitangsu, S. Rasyad, F. Damsi, and A. Silvia, "Real Time Garbage Bin Capacity Monitoring," Comput. Eng. Appl. J., vol. 9, no. 2, pp. 127-133, 2020.

[10] Nyayu Latifah Husni et al, "Garbage Box (G-Box) Designing and Monitoring," ITC CSCC Conf., pp. 58, 2019. 